Copyright (C) 2009 IEEE.

Reprinted from the IEEE MTT-S International Microwave Symposium

Digest (2009 : Boston, MA, USA):pp.245-248

This material is posted here with permission of the IEEE. Such permission of the IEEE does not in any way imply IEEE endorsement of any of the University of Adelaide's products or services. Internal or personal use of this material is permitted. However, permission to reprint/republish this material for advertising or promotional purposes or for creating new collective works for resale or redistribution must be obtained from the IEEE by writing to pubs-permissions@ieee.org.

By choosing to view this document, you agree to all provisions of the copyright laws protecting it. 


\title{
Modeling of Dielectric Material Interfaces for the Radial Point Interpolation Time-Domain Method
}

\author{
Thomas Kaufmann \#, Thomas Merz \#, Christophe Fumeaux *, Rüdiger Vahldieck * \\ \# Laboratory for Electromagnetic Fields and Microwave Electronics - IFH \\ ETH Zurich, Zurich, CH-8092, Switzerland \\ Email: thomas.kaufmann@ifh.ee.ethz.ch \\ * School of Electrical \& Electronic Engineering, The University of Adelaide \\ Adelaide, South Australia 5005, Australia
}

\begin{abstract}
The Radial Point Interpolation Time-Domain (RPITD) method is a flavor of meshless domain discretization methods applicable to computational electromagnetics. Meshless methods do not require an explicit mesh topology, but rather rely on a representation of a physical model as a node distribution. This is firstly advantageous for modeling of conformal boundaries and multi-scale geometries. But as the most attractive feature, the node arrangements can be adapted on-the-fly. The RPITD method is based on interpolation of the field distribution using radial and monomial basis functions. This paper introduces a technique to model arbitrarily shaped dielectric interfaces in the framework of meshless methods. Using the proposed technique, errors associated to the interpolation of non-smooth fields at material interfaces are reduced, as demonstrated for 2D-TE modes. This allows for accurate modeling of interfaces with dielectric contrast. Unlike previous publications which modify the basis functions at interfaces, a physically motivated correction term is introduced here. Errors in the vicinity of material interfaces decrease significantly and simulation accuracy is generally improved.

Index Terms-Meshless Methods, Time-Domain analysis, Radial Point Interpolation.
\end{abstract}

\section{INTRODUCTION}

Meshless methods for computational electromagnetics (CEM) offer promising prospects over classical methods for numerous applications[1]. Replacing the explicit mesh topology by an arbitrarily shaped node distribution opens new perspectives for conformal and multi-scale modeling of electromagnetic problems. Further, adaptation of the node configuration during simulation appears attractive for enhancing the accuracy and for optimization purposes.

In the field of CEM in time-domain, the well-known Smooth Particle Hydrodynamics (SPH) method for fluid dynamics has been adapted to solve Maxwell's equations [2]. The SPH algorithm, nevertheless, requires the application of sophisticated consistency-restoring techniques to achieve sufficient accuracy. Furthermore each node requires a preliminary determined associated volume in the SPH algorithm. The Radial Point Interpolation Method (RPIM) has been introduced as a meshless method in [3] for mechanical analysis. The core algorithm is an interpolation of the field components using radial and monomial basis functions. An adaptation to CEM in time-domain has been introduced by the authors in [4] as Radial Point Interpolation Time-Domain (RPITD) method.

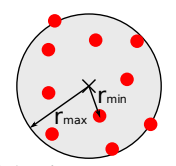

Fig. 1. Support domain with size $r_{\max }$ and minimum distance $r_{\min }$.

The present publication proposes a method for modeling arbitrarily-shaped dielectric interfaces exploiting the unique abilities of meshless algorithms. The discussion is limited here to the lossless 2D-TE case. In the proposed implementation, the node distribution has to be adapted to conform the material interface. Due to the non-continuous property of the electric field at the the material interface, continuous basis functions are not able of accurately interpolating functions with discontinuous first spatial derivatives. This leads to a degradation of the interpolation accuracy expressed by unphysical spatial oscillations in this region. In [5], [6], adaptations of the basis functions were introduced to model discontinuous behavior. In contrast, a physically motivated correction term based on geometrical information is introduced in this article to improve the accuracy of full-wave behavior at the dielectric contrast.

\section{RPITD METHOD}

The RPITD method is a node-based domain discretization simulation technique. It assumes that the field in every node is only influenced by a finite number of nodes in its vicinity. This volume of influence is called support domain (s. Fig. 1). A local interpolation is performed to approximate the value of the fields and their derivatives in the considered node. The interpolated values are used to solve Maxwell's equations in time-domain. The convergence rate for a regular grid distribution is identical to the FDTD method. For unstructured grids it remains to be determined. This paper uses a 2D implementation of a transverse electric (TE) mode. Starting from a given set of nodes where the electric field is evaluated (E-nodes), a Voronoi tessellation [7] is applied to generate a dual grid for the H-nodes. A detailed derivation of the interpolation and update equations is given in [3], [4], and therefore only a short summary of the method is given here.

\section{A. Interpolation}

The local interpolation of the field component $u(\mathbf{x})$ makes use of radial and monomial basis functions and takes the 
following form:

$$
\begin{aligned}
u(\mathbf{x}) \approx\langle u(\mathbf{x})\rangle & =\sum_{n=1}^{N} r_{n}(\mathbf{x}) a_{n}+\sum_{m=1}^{M} p_{m}(\mathbf{x}) b_{m} \\
& =\mathbf{r}^{T}(\mathbf{x}) \mathbf{a}+\mathbf{p}^{T}(\mathbf{x}) \mathbf{b}
\end{aligned}
$$

where $\mathbf{a}$ and $\mathbf{b}$ are interpolation parameters and $\mathbf{r}^{T}(\mathbf{x}), \mathbf{p}^{T}(\mathbf{x})$ are the basis functions evaluated at position $\mathbf{x}=\left(x-x_{c}, y-\right.$ $\left.y_{c}\right)^{T}$ relative to the node to be interpolated. The size of the basis functions is determined by the number of nodes in the support domain $N$ and the order of the monomial basis $M$. The chosen basis functions are of Gaussian (radial) and linear (monomial) type:

$$
r(\mathbf{x})=\exp \left[-c\left(\frac{|\mathbf{x}|}{r_{\max }}\right)^{2}\right], \mathbf{p}^{T}(\mathbf{x})=[1, x, y] .
$$

The shape parameter $c$ controls the width of the basis function. The interpolation operation is done in a preprocessing step and consists in assembling a matrix of basis functions evaluated at the nodes in the support domain. The interpolation parameters $\mathbf{a}, \mathbf{b}$ are then calculated by inversion of this matrix. The size of the matrix and the shape parameter $c$ highly influence both the matrix condition and the accuracy of the interpolation. The interpolation of the spatial derivatives of the field components can be subsequently approximated by using the basis functions and its derivatives:

$$
\begin{array}{r}
\langle u(\mathbf{x})\rangle=\mathbf{r}^{T}(\mathbf{x}) \mathbf{a}+\mathbf{p}^{T}(\mathbf{x}) \mathbf{b}=\mathbf{\Psi}(\mathbf{x}) \mathbf{u}^{\mathbf{e}}, \text { and } \\
\left\langle\partial_{\kappa} u(\mathbf{x})\right\rangle=\partial_{\kappa} \mathbf{r}^{T}(\mathbf{x}) \mathbf{a}+\partial_{\kappa} \mathbf{p}^{T}(\mathbf{x}) \mathbf{b}=\partial_{\kappa} \mathbf{\Psi}(\mathbf{x}) \mathbf{u}^{\mathbf{e}}
\end{array}
$$

with $\partial \kappa$ the spatial derivative in $\kappa=x, y$-direction. This interpolation method amounts to a weighting of the field values at the surrounding nodes $\mathbf{u}^{\mathbf{e}}$ by the shape function $\boldsymbol{\Psi}(\mathrm{x})$. The derivative $\partial_{\kappa} \boldsymbol{\Psi}(\mathbf{x})$ is then used to interpolate spatial derivatives. $\boldsymbol{\Psi}(\mathrm{x})$ and $\partial_{\kappa} \boldsymbol{\Psi}(\mathrm{x})$ are vector functions of length $N$. All operations and matrices are locally defined and (depending on the size of the support domain) have a typical size of $(10 \times 10)$ elements in $2 \mathrm{D}$ computations.

\section{B. 2D-TE Implementation}

The present implementation is restricted for simplicity to a 2D-TE mode with the magnetic fields $H_{x}, H_{y}$ in the propagation plane and a perpendicular electric field $E_{z}$. The starting point is given by the Maxwell equations in time-domain where the electric field is expressed using the electric displacement field, which is best suited do describe the behavior in materials. In this formulation, Faraday's Law contains a term $\nabla \times \frac{D}{\varepsilon}$ for linear materials. At the interface, the permittivity $\varepsilon=\varepsilon(\mathbf{x})$ is a space-dependent value which has to be taken into consideration when calculating spatial derivatives. As an example, the first vector component is expressed as:

$$
\begin{aligned}
\mu \partial_{t} H_{x}=- & \partial_{y} \frac{D_{z}}{\varepsilon}= \\
& \quad-\frac{\partial_{y} D_{z}}{\varepsilon}-D_{z} \partial_{y} \varepsilon^{-1}=-\partial_{y} E_{z}+E_{z} \frac{\partial_{y} \varepsilon}{\varepsilon}
\end{aligned}
$$

The spatial derivatives of the field are approximated by the interpolation functions $\partial_{x, y} \boldsymbol{\Psi}(\mathbf{x})$ and the temporal derivation is solved with an iteration in time using a leapfrog timestepping. At the interface, the spatial derivative of $\varepsilon$ takes the form of a Dirac-type function. A discrete estimation of this derivative is implemented as a correction term $\mathcal{C}$. This yields the following update equations:

H-node $i$ :

$$
\begin{aligned}
& H_{x, i}^{n+\frac{1}{2}}=H_{x, i}^{n-\frac{1}{2}}-\frac{\Delta t}{\mu_{0}} \sum_{k} E_{z, j_{k}}^{n}\left[\partial_{y} \Psi_{k}+n_{y, k} \cdot \Psi_{k} \cdot \mathcal{C}_{k}\right] \\
& H_{y, i}^{n+\frac{1}{2}}=H_{y, i}^{n-\frac{1}{2}}+\frac{\Delta t}{\mu_{0}} \sum_{k} E_{z, j_{k}}^{n}\left[\partial_{x} \Psi_{k}+n_{x, k} \cdot \Psi_{k} \cdot \mathcal{C}_{k}\right]
\end{aligned}
$$

E-node $j$ :

$E_{z, j}^{n+1}=E_{z, j}^{n}+\frac{\Delta t}{\varepsilon_{0} \varepsilon_{r, j}}\left[\sum_{k} H_{y, i_{k}}^{n+\frac{1}{2}} \partial_{x} \Psi_{k}-\sum_{k} H_{x, i_{k}}^{n+\frac{1}{2}} \partial_{y} \Psi_{k}\right]$.

The summation is calculated over all nodes $i_{k}, j_{k}$ within the respective support domain. The correction term $\mathcal{C}$ in (6a), (6b) will be introduced in the subsequent section. It is interpolated at the H-node position using the shape function $\Psi_{k}$ and projected on the normal vector $\mathbf{n}=\left(n_{x}, n_{y}\right)^{T}$ of the dielectric interfaces. The CFL limit is adapted to the conformal node distribution as $\Delta t \leq \min _{i}\left(r_{\min , i} \sqrt{\mu_{0} \varepsilon_{i}}\right)$ and leads to stable simulations.

\section{Dielectric Material Interfaces}

This section details the technique for modeling dielectric interfaces in the RPITD method. In the first part, a concept to conformal modeling of the interface is proposed. Then a correction factor that reduces interpolation errors is introduced using the spatial derivative of the $\mathbf{D}$ field. For simplicity, the discussion is limited to lossless and frequency independent materials are assumed. The treatment of dispersive media would involve convolution operations in the 2D-TE mode implementation which are left out at this point.

\section{A. Node Placement}

The continuity conditions at the interface between two nonmagnetic dielectric materials with permittivities $\varepsilon_{1}$ and $\varepsilon_{2}$ read

$$
\mathbf{E}_{1 \|}=\mathbf{E}_{2 \|}, \mathbf{B}_{1 \perp}=\mathbf{B}_{2 \perp} .
$$

They are weakly enforced by the explicit assignment of nodes at the material interface (Fig. 2) in the case of a 2D-TE mode implementation. Since both the electric and magnetic field in this arrangement are parallel to the direction of their continuity no further steps have to be taken to enforce the continuity conditions. The material properties are modeled as a step function with value $\varepsilon_{1}$ on one side, $\varepsilon_{2}$ on the other side and $\varepsilon_{\text {mean }}=\frac{\varepsilon_{1}+\varepsilon_{2}}{2}$ at the boundary interface. The introduction of this condition is required since the conformal nature of the RPITD method allows the placement of nodes exactly on the interface. This approach leads to a very accurate spatial modeling of the interface. In contrast, the node-based standard FDTD algorithm generally does not allow nodes to be placed at the boundary interface with arbitrary non-rectangular shape. 


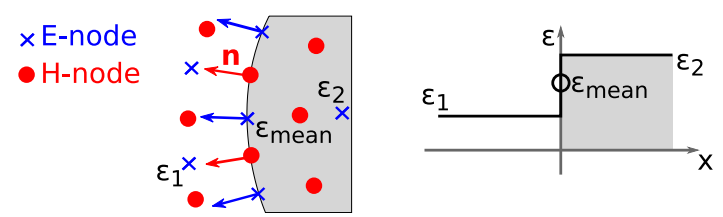

Fig. 2. Node arrangement at material interfaces. The interface is geometrically defined by its normal vector $\mathbf{n}=\left(n_{x}, n_{y}\right)^{T}$ pointing towards the material with a lower permittivity. The material properties at the interface are $\varepsilon_{\text {mean }}=\frac{\varepsilon_{1}+\varepsilon_{2}}{2}$.

\section{B. Discontinuity at the Interface}

At the boundary interface, the electric field is non-zero order discontinuous, i.e. the first spatial derivative is non-continuous. This affects the approximation of the spatial derivative (4). As continuous basis functions only accurately interpolate continuous fields, oscillations of the approximated field can be observed in the vicinity of the discontinuity. This effect is called the Gibbs phenomenon [8]. Literature suggests local adaptations of the basis functions, e.g. by introducing jump functions [5] or by stretching of the radial basis functions [6]. The disadvantage of these approaches are firstly, that the interpolation functions become highly dependent on the geometry, and secondly that material properties have to be incorporated in an otherwise purely mathematical operation.

To circumvent these problems, the spatial derivative of the $\mathbf{D}$ field on the interface is estimated and included into the update equations. Close to the interface, the parallel $\mathbf{D}$ field takes the shape of a step as $\left(\varepsilon_{1}+s(x)\left(\varepsilon_{2}-\varepsilon_{1}\right)\right)$. Thus, the spatial derivative in (5) takes the form of a delta-type function with magnitude $\Delta \varepsilon=\left|\varepsilon_{1}-\varepsilon_{2}\right|$. This leads to the proposed estimation of the correction factor applied in (6):

$$
\mathcal{C}_{k}=\frac{\varepsilon(\mathbf{x})_{\text {estimate }}^{\prime}}{\varepsilon_{\text {mean }}}=\alpha \cdot r_{\text {mean }} \frac{\Delta \varepsilon^{\beta}}{\varepsilon_{\text {mean }}}
$$

with $\varepsilon_{\text {mean }}$ the permittivity on the interface. The factor $r_{\text {mean }}$ is the mean distance to all nodes in the support domain. It introduces a scaling that makes the correction factor independent of discretization. The empirical parameters $\alpha$ and $\beta$ need to be numerically determined by error minimization. It has been numerically confirmed that the behavior of the correction term is independent of the shape parameter $c$ as well as the support domain size $r_{\max }$. This correction factor only applies to nodes with support domain extending over a material interface where $\mathcal{C}_{k}$ takes a non-zero value. The proposed correction term has the advantage of keeping the interpolation operation independent of material properties, while increasing accuracy by a physical approach. This concept is independent of geometry and can be extended to 3D models, which would be more complex to achieve by adapting basis functions.

\section{NUMERICAL EXPERIMENTS}

The numerical experiments presented in the following aims at validating the concept. They investigate the accuracy of the transmission of a plane wave between two materials. First the improvement arising from the correction factor $\mathcal{C}_{k}$ for the modeling of the transmission at normal incidence for different contrasts of permittivities has been quantified. Second, angular incidence is looked at by comparing a regular grid with conformal node distributions. Finally a convergence study is

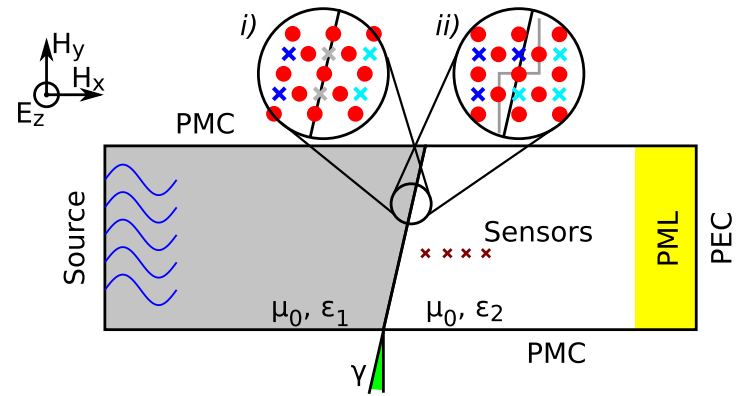

Fig. 3. Simulation model: Incident wave impinges at interface tilted with angle $\gamma$. Dielectric properties on the left hand side are $\mu_{0}, \varepsilon_{1}$ and $\mu_{0}, \varepsilon_{2}$ in the medium on the right hand side. The interface is modeled in two different manners: $i$ ) with a conformal boundary or ii) with a regular grid allowing staircasing effects.

performed to confirm that the error vanishes for increasingly fine spatial discretizations.

All models use harmonic plane waves in a model depicted in Fig. 3. As measure of the accuracy of the interface modeling, the transmission coefficient is recorded and compared with the theoretical expected value:

$$
T_{\perp, t h}=\frac{2 \sqrt{\varepsilon_{1}} \cos \phi_{i}}{\sqrt{\varepsilon_{1}} \cos \phi_{i}+\sqrt{\varepsilon_{2}} \cos \phi_{t}} \text { with } \frac{\sin \phi_{i}}{\sin \phi_{t}}=\sqrt{\frac{\varepsilon_{2}}{\varepsilon_{1}}} .
$$

The absolute error is calculated as the maximum error at several sensor positions as follows:

$$
\text { Error }_{d B}=20 \log \left(\left|T_{\text {num }}-T_{t h}\right|\right) .
$$

For all models, the support domain was set to incorporate $N_{\min } \geq 8$ nodes and the normalized parameter that controls the shape of the radial basis function (2) was set to $c=2$. The optimal parameters in the correction term (8) were found to be $\alpha=47.4, \beta=0.21$.

\section{A. Normal Incidence}

The first numerical analysis is conducted by assessing the numerical error at normal incidence. Contrasts in the range of $\varepsilon_{r}=[1.1,70]$ have been evaluated and the maximum error is depicted at Fig. 4. It can be clearly seen that, especially for higher dielectric contrasts, the correction term significantly increases the simulation accuracy. A decrease in the error of $20 d B$ has been recorded for a high contrast of $1: 70$.

\section{B. Angular Incidence}

In order to determine the influence of conformal modeling of the interface, off-normal incidence has been simulated according to Fig. 3 with various angles $\gamma=\left[0^{\circ}, 7.5^{\circ}\right]$. Since the transmitted wave is propagating in a off-normal direction, the right hand side of the model has been rotated in the direction of the transmitted wave to ensure correct boundary conditions. The conformal material interfaces $(i)$ ) have been compared to a regular grid (ii)). The results of the simulation for a contrast of $10: 1$ at a discretization of $\Delta x=\lambda / 24$ are shown in Fig. 5. As expected the largest error is recorded for a regular grid as staircasing effects degrade the performance. For conformal interfaces, again a significant improvement can be noticed when correction (8) is applied, in comparison with the uncorrected case. The discretization in this numerical experiment is coarser than in the previous 


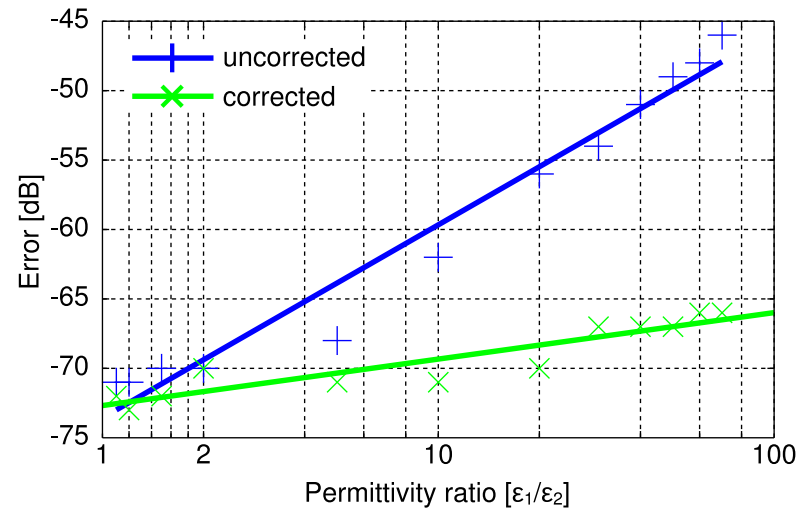

Fig. 4. Error of the transmitted wave amplitude for different contrasts. Comparison between corrected and uncorrected $\left(\mathcal{C}_{k}=0\right)$ approach. Discretization is $\Delta x=\lambda / 30$ at a permittivity of $\varepsilon_{r}=100$.

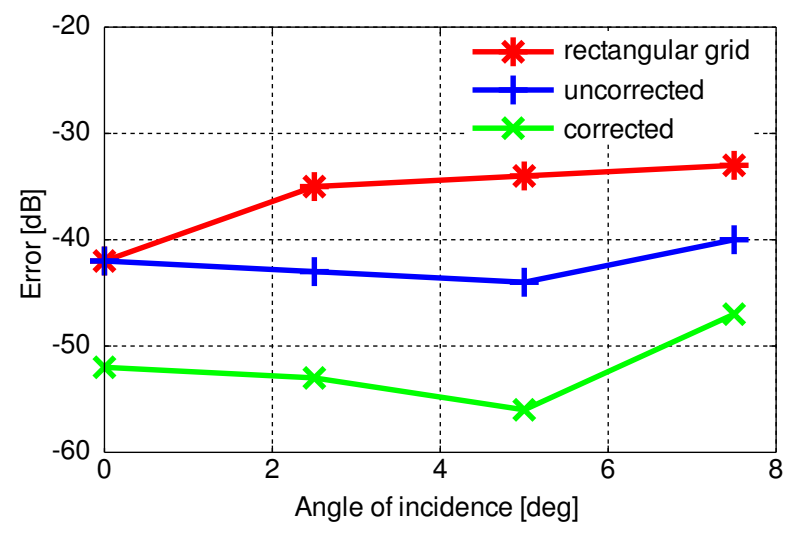

Fig. 5. Error of the transmitted wave amplitude for different angles of incidence, compared with the theoretical value at a contrast of permittivities of $10: 1$ with a discretization of $\Delta x=\lambda / 24$ at $\varepsilon_{r}=$ 10.

results, thus generally the accuracy is degraded compared to that of Fig. 4. Results show that the correction factor also holds for larger angles of incidence. At a contrast of 1:10 and an angle of $\gamma=30^{\circ}$, the numerical error is $-34 \mathrm{~dB}$ for a staggered grid and $-39 \mathrm{~dB}$ (no correction term) and $-42 \mathrm{~dB}$ (correction (8) active) for the conformal grid alignment as determined from preliminary numerical experiments.

\section{Convergence}

A convergence study has been performed for a contrast in permittivity of $50: 1$ at normal incidence. The resolution was increased, starting from a discretization of $\lambda / 21$ to a very fine node arrangement with a spacing of $\lambda / 84$. The numerical experiment has been performed for both the uncorrected and corrected interpolation of the electric field. The results in Fig. 6 show a clear decrease of the error for finer discretizations in both cases, i.e. the error converges towards zero. Additionally it should be expected that, for node distances converging towards zero, both approaches go towards the same solution. This is granted in (8) by the spatial factor $r_{\text {mean }}$ in the correction term $\mathcal{C}_{k}$ which therefore decreases with finer discretization.

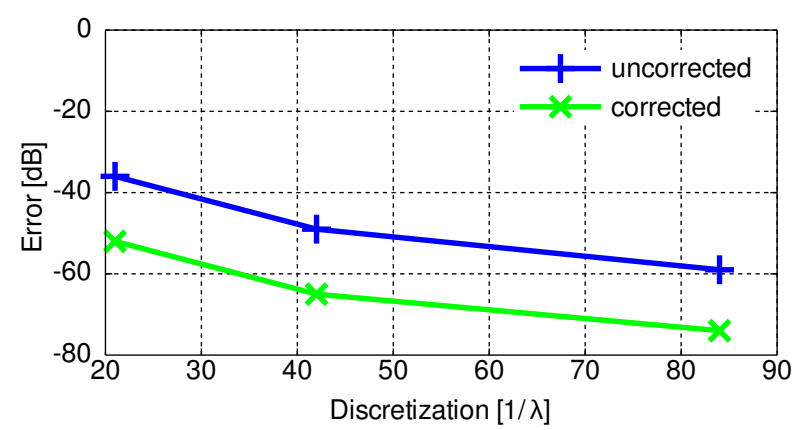

Fig. 6. Convergence study. Clear convergence of the error of the transmission coefficient for both, the corrected and uncorrected approach.

\section{CONCLUSION}

A method for modeling material interfaces for the RPITD method has been introduced. At the interface the approach makes use of the capabilities of conformal node placement of the RPITD method. The interface is modeled by placing nodes on the boundary between two media. Applying the permittivity as a step function determines the value at the interface. Discontinuities of the spatial derivatives of material properties are taken into account through a correction term. This renders the mathematical interpolation operation independent of physical material properties while reducing unphysical field oscillations from the Gibbs phenomenon near interfaces.

Numerical experiments were performed to validate the technique and a significant increase in accuracy has been demonstrated using the proposed correction term. This concept is applicable also to other flavors of meshless domain discretization methods. In contrast to other approaches adapting the basis functions of the interpolation operation, here an extension to a 2D-TM mode or to full-wave analysis in $3 \mathrm{D}$ should be straightforward.

\section{REFERENCES}

[1] G. Liu and Y. Gu, An introduction to meshfree methods and their programming. Dordrecht, The Netherlands: Springer, 2005.

[2] G. Ala, E. Francomano, A. Tortorici, E. Toscano, and F. Viola, "Smoothed particle electromagnetics: A mesh-free solver for transients," Journal of Computational and Applied Mathematics, vol. 191, no. 2, pp. 194-205, 2006.

[3] G. R. Liu and Y. T. Gu, "A local radial point interpolation method (LRPIM) for free vibration analyses of 2-d solids," Journal of Sound and Vibration, vol. 246, no. 1, pp. 29-46, Sept 2001.

[4] T. Kaufmann, C. Fumeaux, and R. Vahldieck, "The meshless radial point interpolation method for time-domain electromagnetics," in IEEE MTT-S Int. Microwave Symposium. Atlanta, GA, USA: IEEE, June 2008, pp. $61-65$.

[5] C. Herault and Y. Marechal, "Boundary and interface conditions meshless methods [for em field analysis]," Magnetics, IEEE Transactions on, vol. 35, no. 3, pp. 1450-1453, May 1999.

[6] R. Gordon and W. Hutchcraft, "Using elliptical basis functions in a meshless method to determine electromagnetic fields near material interfaces," Antennas and Propagation Society International Symposium, 2007 IEEE, pp. 3592-3595, June 2007.

[7] C. B. Barber, D. P. Dobkin, and H. Huhdanpaa, "The quickhull algorithm for convex hulls," ACM Trans. Math. Softw., vol. 22, no. 4, pp. 469-483, 1996.

[8] B. Fornberg, T. A. Driscoll, G. Wright, and R. Charles, "Observations on the behavior of radial basis function approximations near boundaries," Computers \& Mathematics with Applications, vol. 43, no. 3-5, pp. 473 490, 2002. 